\title{
Besondere Herausforderungen der neuen Transparenzbestimmungen
}

\section{Marcel Boller}

Dr. iur., Rechtsanwalt, Zürich

\author{
Nach Inkrafttreten einer neuen Verordnung müssen sämtliche Rabatte im Heilmit- \\ teleinkauf in den Rechnungen und Geschäftsbüchern ausgewiesen werden. Dies \\ soll vor allem dazu dienen, Vergünstigungen im Gesundheitswesen sichtbar zu \\ machen und die Weiterleitung von Rabatten zu fördern. Betroffen von den neuen \\ Bestimmungen sind Ärzte und Apotheker, aber auch Spitäler, Alters- und Pflege- \\ heime, für welche ein Mehraufwand entsteht.
}

Der Bundesrat hat am 10. April 2019 die Verordnung über die Integrität und Transparenz im Heilmittelbereich (VITH) verabschiedet. Diese trat am 1. Januar 2020 in Kraft und konkretisiert die ebenfalls ab dann geltenden Bestimmungen von Art. 55 und Art. 56 des revidierten Heilmittelgesetzes (HMG). Damit gelten im Heilmittelbereich neue Regeln betreffend die Integrität und Transparenz bei entsprechenden Interaktionen zwischen Herstellerinnen, Händlern und Leistungserbringern. Diese neuen Bestimmungen betreffen in besonderem Masse Ärztinnen und Ärzte, da gerade die letzte Handelsstufe im Heilmitteleinkauf im Fokus des Gesetzgebers stand und diesbezüglich neue Pflichten festgesetzt wurden. Weil ein Verstoss gegen die Bestimmungen des revidierten HMG nicht nur verwaltungsrechtlich geahndet werden kann, sondern auch strafrechtlich sanktionsbewehrt ist [1], sollte die Angleichung der eigenen Praxis an die neuen Regeln ein zentrales Gebot darstellen.

\section{Transparenzpflichten}

Seit dem 1. Januar 2020 müssen sämtliche Rabatte im Heilmitteleinkauf in den Rechnungen und Geschäftsbüchern ausgewiesen und auf Verlangen dem Bundesamt für Gesundheit (BAG) offengelegt werden [2]. Der Gesetzgeber beabsichtigte damit, einerseits die Kontrolle und den Vollzug des Verbots geldwerter Vorteile zu stärken und andererseits die Weitergabe der ausgehandelten Rabatte zu verbessern. Die Transparenzpflicht sollte insbesondere auch dazu dienen, Vergünstigungen im Gesundheitsbereich durch die konkrete
Benennung der Rabatte sichtbar zu machen und die Weiterleitung zu fördern.

Von der Transparenzpflicht werden Rabatte und Rückvergütungen im Heilmitteleinkauf erfasst. Unter die Bestimmung fallen nicht nur Arzneimittel, sondern auch Medizinprodukte. Von der Transparenzpflicht ausgenommen sind lediglich freiverkäufliche Arzneimittel (Abgabekategorie E) und klassische Medizinpro-

Da bei den Medizinprodukten ein hoheitlich festgelegter Fabrikabgabepreis fehlt, ist die Festsetzung des Standardpreises nicht immer einfach.

dukte der Klasse I [3]. Durch den Einbezug von Medizinprodukten sind demnach praktisch alle Produkte und Gegenstände mit medizinischem Verwendungszweck in einer Praxis oder einem Spital erfasst. Deshalb dürfte diesbezüglich die Unterscheidung wichtig werden, ob es sich beim eingekauften Medizinprodukt um eines der Klasse I oder eines einer höheren Klasse handelt. Bei letzter Kategorie kommt die Transparenzpflicht stets zur Anwendung, was eine sorgfältige Buchführung über die Rabatte bedingt.

Gemäss der Definition in der VITH gilt als Rabatt die Differenz zwischen dem Standardpreis und dem effektiv bezahlten Preis für ein konkretes Produkt [4]. Wichtig für die Leistungserbringer ist es daher, dass solche Rabatte beim Einkauf von Medizinprodukten identifiziert und transparent ausgewiesen werden. Da bei den Medizinprodukten ein hoheitlich festgelegter Fabrikabgabepreis fehlt, dürfte die Festsetzung des Standardpreises nicht immer einfach sein. In praktischer Hinsicht bleibt 
aber wohl keine andere Wahl, als die von den Herstellern verwendeten Listenpreise als Norm aufzufassen und jede Abweichung davon als Rabatt zu betrachten. In persönlicher Hinsicht kommt die Transparenzpflicht in allen Konstellationen zur Anwendung, in denen Rabatte oder Rückvergütungen an Personen oder Organisationen gewährt werden, die Heilmittel verschreiben, abgeben, anwenden oder zu diesem Zweck einkaufen [5]. Demnach haben diese Pflicht auf Einkaufsseite die Spitäler, Alters- und Pflegeheime, Ärzte und Apotheker zu beachten. Auf Seite der Verkäufer werden insbesondere die Hersteller, Vertriebsfirmen, Grosshändler und Detailhandelsgeschäfte zur Dokumentation verpflichtet. Gemäss den Erläuterungen zur VITH zielt die Transparenzpflicht auf die letzte Handelsstufe beim Heilmitteleinkauf, weshalb die Leistungserbringer als Einkäufer unter besonderem Fokus stehen. Diese haben die Rabatte sowohl beim Einkauf bei Herstellern als auch beim Bezug über Grosshändler als solche auszuweisen.

Es ist allerdings jeweils im Einzelfall zu prüfen, ob Handlungen, welche Handänderungen bei Arzneimitteln oder Medizinprodukten beinhalten, gemäss der Intention des Gesetz- und Verordnungsgebers unter die Transparenzpflicht fallen. Konstellationen, in welchen Arzneimittel nicht im Rahmen des Einkaufs weitergegeben werden, dürften tendenziell davon nicht erfasst werden. Zu denken ist hier etwa an die Abgabe der Musterpackungen [6] oder Rabattierungen bzw. Rückvergütungen im Rahmen der Einzelfallvergütung [7].

\section{Weitergabepflichten}

Die Transparenzpflicht weist einen engen Konnex zu den Weitergabepflichten der Leistungserbringer aus.

\section{Das Wichtigste in Kürze}

- Zwei neue Artikel über Integrität und Transparenz ersetzen den bisherigen Art. 33 LPTh über die Zusage und Annahme materieller Vorteile. Die neue Verordnung über die Integrität und Transparenz im Heilmittelbereich (OITPTh) regelt die Details.

- Diese neuen Bestimmungen betreffen insbesondere Ärzte, da sich der Gesetzgeber auf die letzte kommerzielle Phase des Kaufs konzentriert hat. Seit dem 1. Januar 2020 müssen alle Rabatte beim Kauf von Heilmitteln auf Rechnungen und Handelsbroschüren ausgewiesen und auf Wunsch dem Bundesamt für Gesundheit (BAG) mitgeteilt werden.

- Zudem besteht höchstens eine Verpflichtung zur Übermittlung der Daten nach Art. 56 KVG/LAMal. Daher müssen die Ärzte alle Rabatte in allen Geschäftsbeziehungen identifizieren und gegebenenfalls an Versicherungen oder Patienten weitergeben.
Die Leistungserbringer unterstehen bereits heute einer Weitergabepflicht. Leistungserbringer müssen die direkten und indirekten Vergünstigungen, die ihnen bei der Lieferung von kassenpflichtigen Arzneimitteln und Medizinprodukten gewährt werden oder die ihnen ein anderer in ihrem Auftrag tätiger Leistungserbringer gewährt, an die versicherte Person bzw. an den Versicherer weitergeben [8]. Die Weitergabepflicht erstreckt sich ebenfalls auf Vergünstigungen auf Verbrauchsmaterialien oder Geräten, welche für die Untersuchung oder Behandlung eingesetzt werden.

Ein besonderes Augenmerk gilt indes den gleichzeitig revidierten Artikeln in der Verordnung über die Krankenversicherung (KVV) [9]. Darin werden die Einzelheiten bzw. die Ausnahmen der grundsätzlichen Weitergabepflicht festgelegt. Demnach haben die Leistungserbringer die erhaltenen Rabatte weiterzugeben, wobei die Pflicht zur direkten Weitergabe grundsätzlich auf den ambulanten Behandlungsbereich beschränkt ist [10]. Die Leistungserbringer dürfen allerdings bis zu 49\% der gewährten Vergünstigungen einbehalten, sofern zwischen Versicherer und Leistungserbringer eine entsprechende Vereinbarung geschlossen wurde [11]. Dabei wird vorausgesetzt, dass die nicht weitergegebenen Vergünstigungen nachweislich zur Verbesserung der Qualität der Behandlung eingesetzt werden. Betreffend diese Vereinbarungen zwischen Versicherern und Leistungserbringer bestehen allerdings noch einige Unsicherheiten insbesondere in Bezug auf Regelungsform und -tiefe. Um den administrativen Aufwand zu reduzieren, werden diese Vereinbarungen in der Regel zwischen den Verbänden abgeschlossen.

Der Grundsatz der Weitergabe von Rabatten bestand zwar bereits bisher, aber die Umsetzung und Durchset-

\section{L'essentiel en bref}

- Deux nouveaux articles relatifs à l'intégrité et à la transparence remplacent l'ancien art. 33 LPTh sur la promesse et I'acceptation d'avantages matériels. La nouvelle ordonnance sur l'intégrité et la transparence dans le domaine des produits thérapeutiques (OITPTh) règle les détails.

- Ces nouvelles dispositions touchent particulièrement les médecins, puisque le législateur s'est concentré sur la dernière étape commerciale de l'achat. A partir du $1^{\text {er }}$ janvier 2020 , tous les rabais à l'achat de produits thérapeutiques devront figurer sur les factures et les livrets commerciaux et, sur demande, être communiqués à I'Office fédéral de la santé publique (OFSP).

- En outre, il existe tout au plus une obligation de transmettre les données conformément à l'art. 56 LAMal. Par conséquent, les médecins doivent identifier tous les rabais dans toutes les relations d'affaires et, le cas échéant, les répercuter sur les compagnies d'assurance ou les patients. 
zung der geltenden Bestimmung erwies sich in der Praxis als äusserst schwerfällig. Indem nun die Vollzugskompetenzen beim BAG gebündelt werden, ist zu erwarten, dass die entsprechenden Bestimmungen mehr Popularität erhalten werden. Im Handel mit Arzneimitteln ist die Kenntnis dieser Weitergabepflicht deshalb wichtig, weil die Weitergabe einen Einfluss auf die Einhaltung des Vorteilsverbots haben kann [12]. Werden Rabatte weitergegeben, so ist ein gleichzeitiger Verstoss gegen den Integritätsgrundsatz wohl ausgeschlossen, da der Vorteil nicht beim Leistungserbringer, sondern bei der Versicherung bzw. beim Patienten anfällt. Entsprechend werden in den künftigen Vertriebsverträgen mit Herstellern bzw. Grossisten häufig entsprechende Klauseln zu finden sein, welche eine Bestätigung der Einhaltung von Art. 56 KVG verlangen.

\section{Besondere Herausforderungen}

Aus den Erläuterungen des Bundesrats geht hervor, dass seit 1. Januar 2020 sämtliche Formen der finanziellen Unterstützung von Leistungserbringern beim Heilmitteleinkauf von der Transparenz- bzw. Weitergabepflicht erfasst werden. Es entstehen daher neue Verpflichtungen für Ärztinnen und Ärzte, da die Bestimmungen insbesondere auf die letzte Handelsstufe zielen. Leistungserbringer müssen darauf achten, dass Rabatte und Rückvergütungen auf Arzneimitteln und Medizinprodukten transparent ausgewiesen und gemäss Art. 56 KVG weitergegeben werden. Vergünstigungen können nicht nur im Sinne von direkt ausgewiesenen Rabatten auf einzelnen Lieferungen, sondern auch mittels umsatzabhängiger Rückvergütungen oder Koppelungsgeschäften vermittelt werden. Es genügt demnach nicht, bei der Umsetzung der revidierten Bestim- mungen nur die direkten Rabatte zu analysieren. Vielmehr sollten auch Absprachen und Modelle einbezogen werden, bei denen Heilmittel zu ausserordentlich günstigen Konditionen eingekauft werden. Auch in solchen Konstellationen gilt die Transparenzpflicht nach Art. 56 HMG. Selbst indirekt gewährte Rabatte, die eine Reduktion des Standardpreises bedeuten, müssen in den Geschäftsbüchern als solche ausgewiesen werden.

Damit entsteht bei den Ärztinnen und Ärzten naturgemäss ein Mehraufwand, da die Identifikation dieser Rabatte, wenn es sich nicht um einfach gelagerte Einkäufe handelt, schwierig sein dürfte. Ausserordentlich günstige Angebote oder Koppelungsgeschäfte sollten jedenfalls genauer untersucht werden. Der Gesetz- und der Verordnungsgeber nahmen diesen Mehraufwand aber wohl in Kauf, da die Revision hauptsächlich auf mehr Transparenz und auf damit verbundene Einsparungen zielt. Der Vorschlag des Bundesrats, einen allfälligen Zusatzaufwand der Leistungserbringer bei der Umsetzung der Bestimmungen vor der Weitergabe der Rabatte abzugsfähig zu machen, wurde jedenfalls während der Ausarbeitung der VITH wieder gestrichen. In diesem Sinne ist auch in der künftigen Praxis nicht mit (grosszügigen) Ausnahmen zu rechnen.

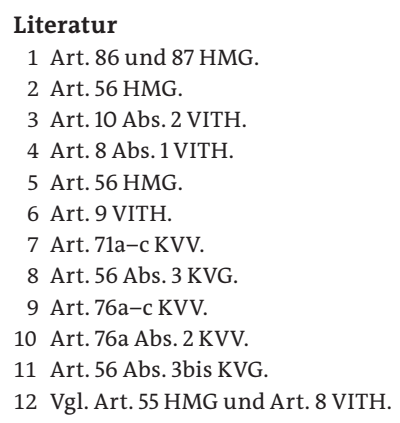

